MATEC Web of Conferences 22,01005 (2015)

DOI: $10.1051 /$ matec conf/ 20152201005

(C) Owned by the authors, published by EDP Sciences, 2015

\title{
Review of Key Technologies of 5G Wireless Communication System
}

\author{
Sha Shi*, Wujun Yang, Jirong Zhang \& Zhixian Chang \\ School of Communication and Information Engineering, Xi' an University of Posts and Telecommunications, \\ Xi'an, Shaanxi, China
}

\begin{abstract}
The 5th generation mobile communication system $(5 \mathrm{G})$ is oriented towards a new generation of mobile communication system to the year of 2020 and beyond, and its development is still at the exploratory stage. Combining the latest trends in mobile communication development at home and abroad, in this article, we describe the key technologies of driving the 5G research direction. Furthermore, the technical innovation of 5G comes from both wireless and network technologies. In the field of wireless technologies, massive multiple-input multiple-output (MIMO), ultra-wideband spectral, ultra-dense heterogeneous networks, have already become the focus of global industry. In the field of network technologies, a new network architecture based on software-defined networking (SDN) becomes the prevailing view worldwide. Additionally, there are some other potential technologies for 5G, such as NOMA, FBMC, mm Waves, and Multi-carrier technology aggregation.
\end{abstract}

Keywords: 5G, the key technologies, wireless technologies, network technologies

\section{INTRODUCTION}

Mobile communications have profoundly changed in everyday life, and people's desire for higher-performance mobile communications is never ending. In the history of mobile communications, each generation can be defined by some key technologies. For example, $1 \mathrm{G}$ system uses Frequency Division Multiple Access (FDMA) and can provide analog voice service only. $2 \mathrm{G}$ system is mainly based on Time Division Multiple Access TDMA and can provide both digital voice and low data-rate services. $3 \mathrm{G}$ system, marked by Code Division Multiple Access (CDMA), can achieve the peak data rates from 2 Mbps to tens of Mbps, and support multimedia services. On the basis of OFDMA technology, 4G system can achieve the peak data rates from $100 \mathrm{Mbps}$ to $1 \mathrm{Gbps}$, and support various mobile broadband (MBB) services. Going forward, following the large-scale commercialization of $4 \mathrm{G}, 5 \mathrm{G}$ is expected to be commercialized towards the year of 2020 and beyond, has become a focal point for global research and development [1]. However, 5G is not a revolution but an evolution. As far as we are concerned, there will not be great changes in the core network, and the key technologies are in the wireless part [2]. In this paper following, several technologies are discussed for $5 \mathrm{G}$ service delivery. The objective is to meet a variety of application scenarios, to achieve better quality of experience (QoE). The paper is organized as follows. In Section 2, wireless technologies for $5 \mathrm{G}$ are reviewed. The corresponding network technology is outlined in Section 3. The other potential technologies are described in Section 4.

*Corresponding author:ssha 5g@163.com

\section{5G WIRELESS TECHNOLOGIES}

\subsection{Massive MIMO}

MIMO has extensively applied in WIFI、 LTE and so on. In theory, the more antennas, the higher spectral efficiency and transmission reliability. In particular, when the number of transmit antennas and receive antennas are large, MIMO channel capacity will linearly increase approximately with the number of antennas in the minimum value. Therefore, with a large number of antennas and this provides an efficient pathway to greatly improve the capacity of the system. Due to limitations of space occupied by the multi-antenna, the implementation complexity of technical conditions, in the current wireless communication systems, the number of antenna transceiver multi-side configuration is not large, for example, the LTE system uses a maximum of four antennas, LTE-A system uses up to eight antennas [3]. However, because of its enormous capacity and reliability gains, research related technology for large number of MIMO antenna system attracts the attention of researchers, such as in a cell, the research of multi user MIMO system which the base station equips with greatly exceed the number of antenna of mobile station's [4].

Massive MIMO can be achieved by some inexpensive and low power components. It makes a wide prospect in high frequency to implement mobile communication. There are some advantages such as increasing exponentially wireless spectrum efficiency, enhancing network coverage and system capacity, helping operators maximize the use of existing sites and spectrum resources [5].

3D-MIMO adds vertical dimension on the basis of MIMO, see Fig.1. So it makes three-dimensional beam forming in space, and avoids the mutual inter- 
ference [6]. We can achieve multi-directional beam forming with massive MIMO.

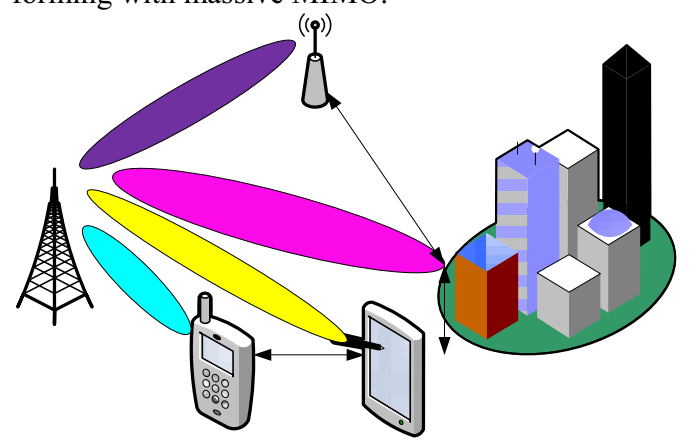

Figure1. 3D-MIMO architecture

\subsection{Non-Orthogonal Multiple Access, NOMA}

We know 3G adopts Direct Sequence CDMA (DS-CDMA) technology, the receiving end uses rake receiver, and due to its non-orthogonal feature, we have to use fast power control (Fast Transmission power Control, TPC) to solve the distance effect.

4G adopts OFDM technology, which can overcome multipath interference and most importantly it combines with MIMO, which makes user data higher [7] Due to multi-users are orthogonal, there is no distance effect, so TPC is discarded, and AMC is adopted to achieve the link adaptation.

NOMA hopes to regain the non-orthogonal multi-users multiplexing principle of $3 \mathrm{G}$, and integrate it in the 4G OFMA technology.

From $2 \mathrm{G}, 3 \mathrm{G}$ to $4 \mathrm{G}$, multi-users multiplexing technologies are time domain, frequency domain and code domain; whereas NOMA is from power domain, see Fig.2.The purpose of this new domain of power is to use a different path loss for each user to achieve multi-user multiplexing.

To achieve multi-user multiplexing in power domain, Sustained Interference Cancellation (SIC) is needed. We can distinguish different users information through the SIC and channel coding, seeFig.3.

NOMA can superpose the transmission signal of multi-channel by using different path loss difference, so that increasing signal gains. It allows all mobile devices in the same cell coverage may able to get the maximum access bandwidth, and we can solve network challenge which brings about large-scale connection.

Also NOMA has its advantage: we need not know the channel state information (CSI), and can get better performance at high-speed moving scenes, organizing better backhaul mobile node links.

\subsection{Ultra-Wideband Spectral}

Channel capacity and bandwidth are proportional to signal to noise ratio (SNR).In order to meet $5 \mathrm{G}$ network Gbps level data rate, the greater bandwidth is needed.

The higher frequency, the greater bandwidth and channel capacity. Therefore, continuous high frequency bandwidth has become an inevitable choice for $5 \mathrm{G}$.

Thanks to some technologies of enhancing the spectral efficiency (such as: massive MIMO), we can achieve $10 \mathrm{Gbps}$ transfer rates in the $1 \mathrm{GHz}$ super bandwidth

\begin{tabular}{|c|c|c|c|}
\hline & $3 \mathrm{G}$ & $3.9 \mathrm{G} / 4 \mathrm{G}$ & $5 \mathrm{G}$ \\
\hline $\begin{array}{l}\text { multi-users } \\
\text { multiplexing }\end{array}$ & $\begin{array}{c}\text { Non-orthogonal } \\
\text { (CDMA) }\end{array}$ & $\begin{array}{l}\text { Orthogonal } \\
\text { (OFDMA) }\end{array}$ & $\begin{array}{l}\text { Non-orthogonal with } \\
\text { SIC(NONA) }\end{array}$ \\
\hline $\begin{array}{c}\text { Signal } \\
\text { waveform }\end{array}$ & Single carrier & $\begin{array}{c}\text { OFDM } \\
\text { (Or DFT-s-OFDM) }\end{array}$ & $\begin{array}{c}\text { OFDM } \\
\text { (Or DFT-s-OFDM) }\end{array}$ \\
\hline $\begin{array}{c}\text { Link } \\
\text { Adaptation }\end{array}$ & Fast TPC & AMC & $\begin{array}{c}\text { AMC+Power } \\
\text { allocation }\end{array}$ \\
\hline \multirow{4}{*}{ Shape } & $\begin{array}{l}\text { Non-orthogonal } \\
\text { assisted by power } \\
\text { control }\end{array}$ & $\begin{array}{c}\text { Orthogonal between } \\
\text { users }\end{array}$ & \multirow[t]{2}{*}{$\begin{array}{c}\text { Superposition } \$ \text { power } \\
\text { allocation }\end{array}$} \\
\hline & & & \\
\hline & $\longrightarrow$ & & $\mathrm{F}$ \\
\hline & & & \\
\hline
\end{tabular}

Figure 2. Comparison with $3 \mathrm{G}, 4 \mathrm{G}$, and5G Multi-users multiplexing technologies 


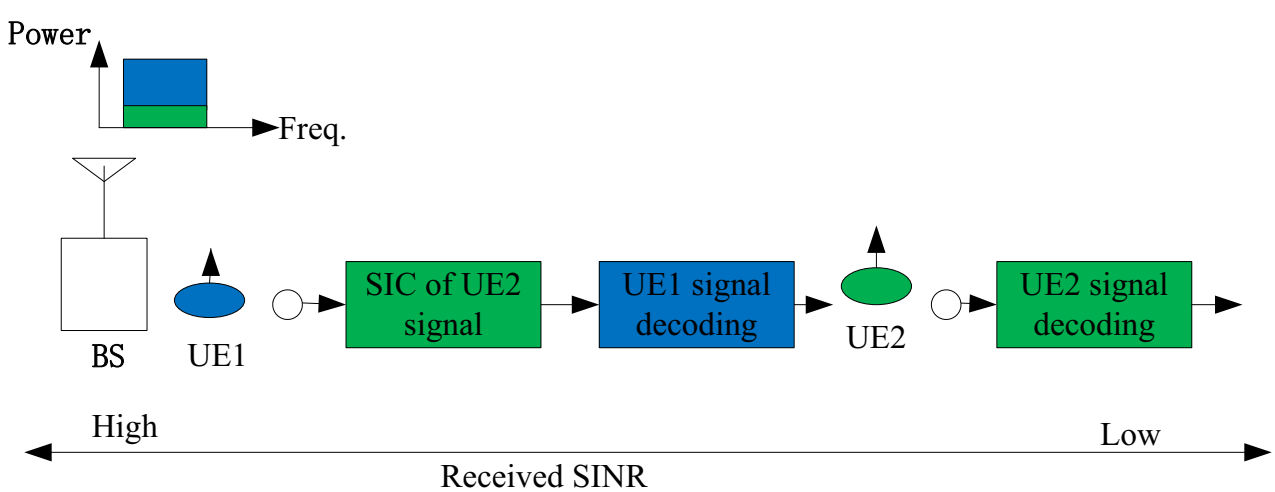

Figure 3. NOMA principle of UE receiving terminal using SIC

All-spectrum access can exploit a variety of spectrum resources for mobile communications, including high and low, paired and unpaired, licensed and unlicensed, contiguous and non-contiguous frequency bands, to increase data rates and system capacity. The frequency bands below $6 \mathrm{GHz}$ are preferred due to their good propagation characteristics. The abundant unused spectrum resources between $6 \mathrm{GHz}$ and 100 $\mathrm{GHz}$ can serve as supplementary bands of 5G.The main challenges of all-spectrum access include channel measurement and modeling, unified access for low frequency and high-frequency bands, unified high-frequency access \& backhaul, and implementation of radio frequency $(\mathrm{RF})$ components.

\subsection{Ultra-dense Heterogeneous Network}

Heterogeneous network is to lay a large number of microcells, picocells, femtocells and other access points in the macro cellular network, in this condition; it can meet the growth requirements of data capacity [8].

In the ultra-dense heterogeneous networks, network densification makes network node closer to the terminal,which brings improvement of power efficiency, spectral efficiency, and system capacity, as well as businesses between a variety of access technologies and various coverage levels sharing flexibility. While ultra-dense heterogeneous networks show a bright future, due to the reduced distance between the nodes and it will cause some problems which are different from the existing system. In the $5 \mathrm{G}$ network, there may be co-channel interference between the same kind of radio access technology deployments, the share spectrum interference between different wireless access technologies, interference between the different levels of coverage. How to solve the property damage caused by interference, to achieve a variety of wireless access technologies, multi-level coverage coexistence, which is an important issue that needs to further study [9-10]; due to the different requirements of different services and QoS (quality of service), different services in the network share [11-12], collaborative strategies between the various nodes, and the network selection [13] is a key issue to ensure the system performance. In order to achieve large-scale collaborative nodes, a large number of neighboring nodes are needed [14]; since more cell boundaries, more irregular, leading to more frequent and more complex handover; it is difficult to ensure the mobility performance. Therefore, the need for ultra-dense network scenarios is to develop new handover algorithm [15]; in order to maximize the network speed, the use of radio waves propagation path loss characteristics, proposed heterogeneous networks and multi-user shared space magnitude proactive interference cancellation method [16]; based on the proposed collaborative 3D multi-point transmission (coordinated multiple points transmission / reception, CoMP) method, to solve the problem of Intercell interference [17], improve the cell edge UE (user equipment) performance, while without any signaling overhead, but the new antenna must be configured in a base station; the method described above is the use of two cells cooperation to solve the interference problems. But the future $5 \mathrm{G}$ networks and multiple networks coexisting, there may be multi-cell collaboration. What's more, with increasingly scarce spectrum resources, it may not have enough band to be assigned, so the future proposed interference cancellation method should be possible to improve resource efficiency. Enhancing capacity by ultra-dense heterogeneous deployment is the most intuitive way, and also domestic and foreign research organizations are focused on objects.

As far as we are concerned, hundreds of billions of devices to connect to a network creates a true "Internet of Everything". This will give rise to emerging industries of unprecedented scale and instill infinite vitality to mobile communications. Meanwhile, the massive amount of interconnected devices will also pose new challenges to mobile communications.

\section{5G NETWORK TECHNOLOGIES}

SDN is a new approach in networking technology, designed to improve the complex and static nature of 


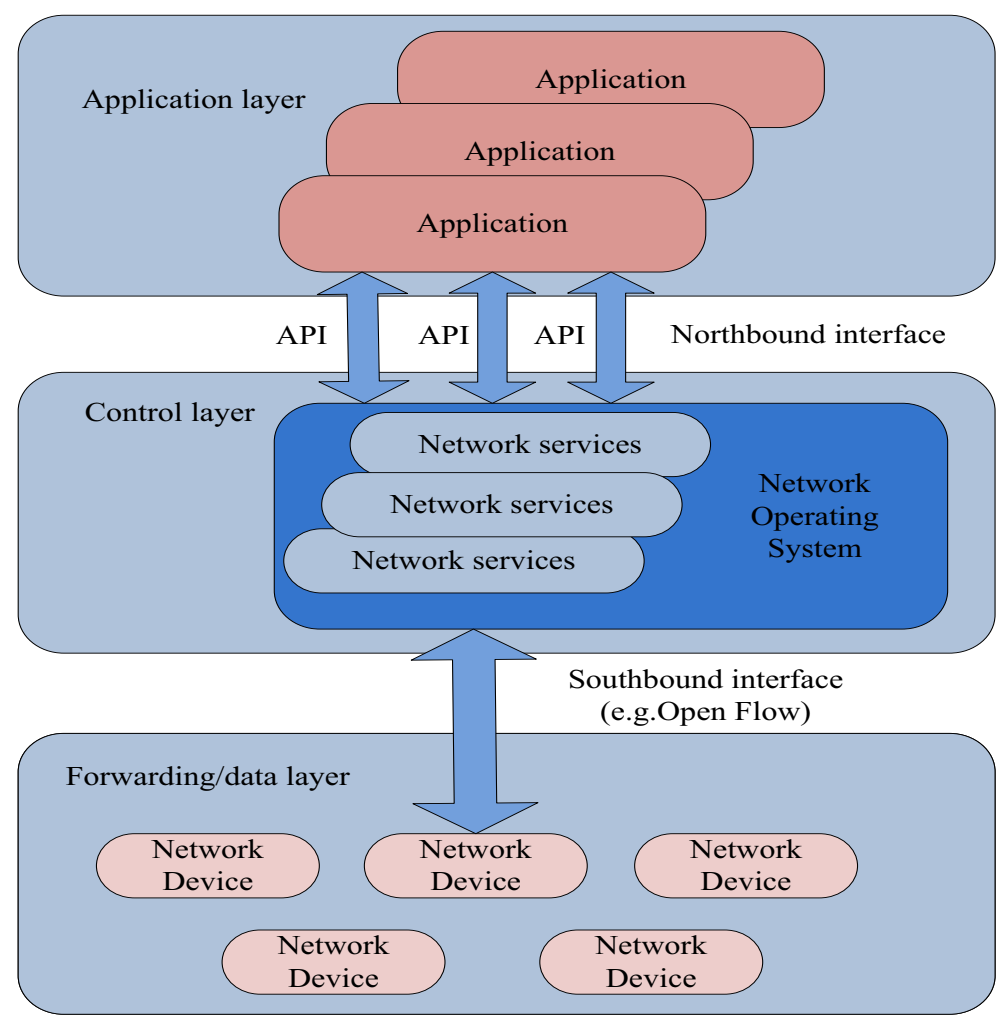

Figure4. SDN architecture

traditional distributed network architectures. The SDN concept is based on split architecture where control plane is separated from underlying physical infrastructures [18-19]. Independent and centralized unit to control the network is provided. Since all control functions are moved to a programmable controller, administrators can easily alter network behavior in real-time and adapt it to quickly-changing network conditions by running appropriate controller applications. Three-layer model that SDN uses is illustrated in Fig.4. Communication between different layers is realized through two interfaces: (a) the northbound interface which connects applications to the controller (REST, JSON, XML, etc.) and (b) the southbound interface which handles all communications between controller and data-forwarding plane [20]. So far, Open Flow protocol is the most commonly used southbound interface.

\section{5G SOME OTHER POTENTIA TECHNOLO- GIES}

\subsection{Filter Bank Multi Carrier (FBMC)}

In OFDM, each subcarrier is orthogonal in time domain, their frequency spectra overlapping and thus it has higher spectrum utilization. OFDM technology is generally used in a wireless data transmission system. In OFDM, there is inter-symbol interference (ISI) just as multi-path in wireless channel. In order to eliminate ISI, the guard interval is inserted. The simple method is setting zero between symbols, i.e. let a period of time when sending the first symbol, and then sending the next one. While it weakens or eliminates the ISI, due to the destruction of orthogonality between subcarriers, it leads to interference (ICI) between subcarriers. Therefore, this method can not be used in an OFDM system. In order to eliminate ISI as well as ICI, cycle prefix is on generally [21]. CP is a system overhead; the effective data is not transmitted, thereby reducing the spectral efficiency. We can see from Fig.5. FBMC achieves multi-carrier transmission by a set of no overlapping band limited sub-carrier. FMC for the inter-carrier frequency offset is very small due to interference, no CP (Cyclic Prefix), greatly improving the efficiency of the frequency [22].

FBMC attracts more and more people's research interest which is an important choice as a multicarrier scheme to $5 \mathrm{G}$ system [23-27]. In FBMC, multi-carrier performance depends on the design of the prototype filter and modulation filter and in order to meet demands of the specific frequency response characteristics, the length of the prototype filter is far greater than the number of subchannels. The implementation complexity is high and not conducive to the realization of hardware. Therefore, the requirements of $5 \mathrm{G}$ filter algorithm is an important research content of FBMC technology in line with the development. 


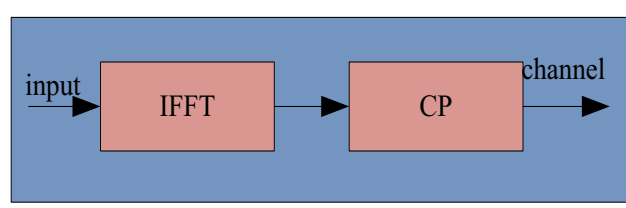

OFDMA

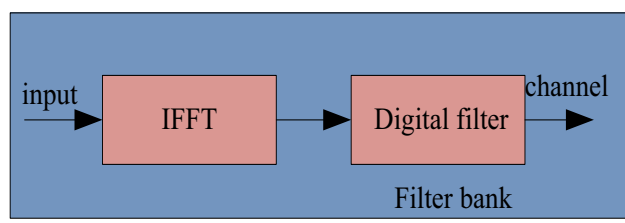

FBMC

Figure 5. Up: the OFDM system. Down: the FBMC technology

\subsection{Millimeter waves, mmWaves}

MmWaves is from $30 \mathrm{GHz}$ to $300 \mathrm{GHz}$ in frequency, 10 millimeter to 1 millimeter in wavelength. MmWaves technology can support ultrahigh speed of transmission rate, narrow beam, flexible and controllable, and it can connect many devices.

Considering the example illustrated in Fig.6.The blue phone is in the edge of $4 \mathrm{G}$ cellular, and some barriers such as buildings (houses) and so on, at this time, we can use the millimeter - wave transmission, bypassing the building blocks to achieve high-speed transmission. Similarly, pink phones can also be implemented the connection to the $4 \mathrm{G}$ cellular using millimeter-wave, without interference. Of course, as the green one is closer to the $4 \mathrm{G}$ cellular, we can connect directly to it.

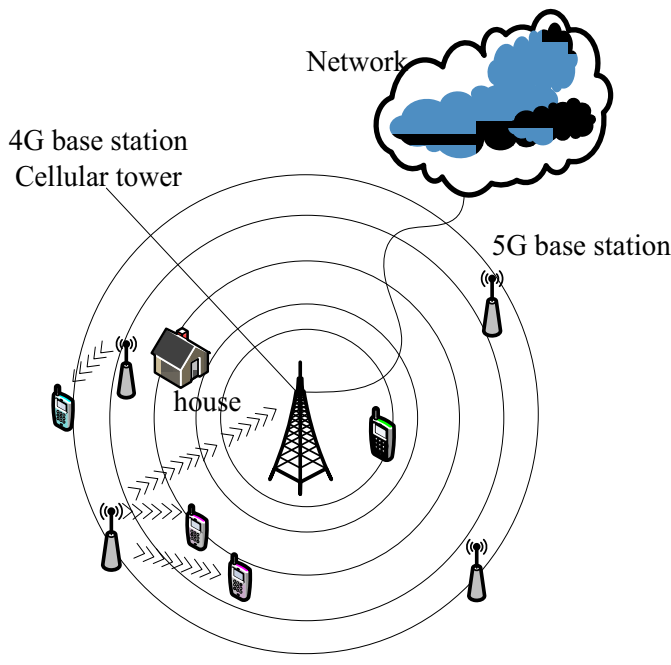

\subsection{Multi-carrier technology aggregation}

Future network is an integrated network, Fig.7.shows the contiguous and non-contiguous carrier aggregation. 5 contiguous $20 \mathrm{MHz}$ band into a polymerization 100 $\mathrm{MHz}$ bandwidth, 5 non-contiguous $20 \mathrm{MHz}$ into a polymerization $100 \mathrm{MHz}$ bandwidth. Besides, it not only achieves the polymerization within the LTE inter-carrier, but also extends to fusion with $3 \mathrm{G}$, WIFI and so on.
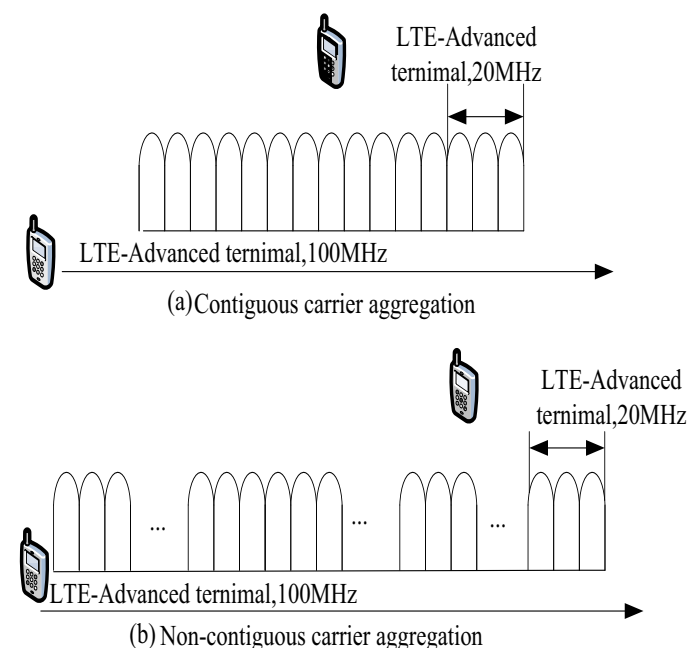

Figure 7. Model of Multi-carrier technology aggregation

Multi-carrier aggregation technology together with heterogeneous network will eventually achieve seamless connection between all things.

\section{CONCLUSION}

In this paper, we discuss some key technologies for $5 \mathrm{G}$ wireless communication system. According to the development law of mobile communications, 5G technology will be commercially available towards 2020 and beyond, its basic goal is to satisfy the demand of the rapid growth in mobile Internet services which bring new business experience. Now the research of $5 \mathrm{G}$ technologies are still at the exploratory stage, and the next few years will determine their technology needs, key indicators and enabling technologies critical period. With the deepening of the research, 5G key supporting technologies will gradually be clear, and enter a substantive stage of standardization research and development in the coming years.

Figure6. The example of mmWaves transmission 


\section{ACKNOWLEDGEMENT}

This work is supported by Natural Science Foundation of Education Department of Shaanxi Province, China (No.2014JM8320) and Industrial research project of S cience and Technology Department of Shaanxi Provin ce(2014K09-14) and International cooperation project of Shaanxi province science and Technology Departm ent(2014KW02-02)

\section{REFERENCES}

[1] $5 \mathrm{G}$ vision and requirements of $5 \mathrm{G}$ forum, Korea, presented at the ITUR 2020 Vision Workshop 5G Forum Korea, Ho Chi Minh, Vietnam, Feb. 2014

[2] Wang C X, Haider F. \& Gao X Q, et al. 2014. Cellular architecture and key technologies for $5 \mathrm{G}$ wireless communication networks. IEEE Commun. Mag., 52: 122-130.

[3] 3GPP. 2010. Physical Channels and Modulation (Release 11). 3GPP TS36.211.

[4] Marzetta T L. 2006. How Much training is required for multiuser MIMO? In: Proceedings of the 40th Asilomar Conference on Signals, Systems, \& Computers, Pacific Grove, 359-363

[5] V. Jungnickel et al. 2014. The role of small cells coordinated multipoint, and massive MIMO in 5G, IEEE Communications Magazine, 52(5).

[6] Frederick W.Vook, Amitava Ghosh. \&Timothy A Thomas. MIMO and Beamforming Solutions for 5G Technolog. Microwave Symposium(IMS), 2014 IEEE MTT-S International, pp: 1-4

[7] W. H. Chin, Z. Fan, and R. Haines, 2014. Emerging technologies and research challenges for $5 \mathrm{G}$ wireless networks, IEEE Wireless Commun., 21: 106-112.

[8] Osseiran A, Braun V, Hidekazu T, et al. 2013. The foundation of the mobile and wireless communications system for 2020 and beyond: Challenges, enablers and technology solutions. In: Proceedings of IEEE Vehicular Technology Conference (VTC Spring), pp: 1-5

[9] Galiotto C, Marchetti N. \& Doyle L. 2012. Flexible spectrum sharing and interference coordination for low power nodes in heterogeneous networks. In: Proceedings of IEEE Vehicular Technology Conference (VTC Fall), pp: $1-5$.

[10]Dhillon H S, Ganti R K. \& Baccelli F, et al. 2012. Modeling and analysis of K-tier downlink heterogeneous cellular networks. IEEE J. Sel. Area Commun., 30: 550-560.

[11]Amani M, Aijaz A. \& Uddin N, et al. 2013. On mobile data offloading policies in heterogeneous wireless networks. In: Proceedings of IEEE Vehicular Technology Conference (VTC Spring), pp: 1-5.

[12] Aijaz A, Aghvami H. \& Amani M. 2013. A survey on mobile data offloading: Technical and business perspectives. IEEE Wirel. Commun., 20: 104-112.

[13] Tabrizi H, Farhadi G. \& Cioffi J. 2011. A learning-based network selection method in heterogeneous wireless systems. In: Proceedings of IEEE Global Telecommunications Conference (GLOBECOM), pp: 1-5.

[14] Prasad A, Lunden P. \& Tirkkonen O, et al. 2013. Mobility state based flexible inter-frequency small cell discovery for heterogeneous networks. In: Proceedings of IEEE the International Symposium on Personal, Indoor and Mobile Radio Communications (PIMRC), pp: 2057-2061.

[15]Lopez-Perez D, Guvenc I. \& Chu X L. 2012. Mobility enhancements for heterogeneous networks through interference coordination. In: Proceedings of IEEE Wireless Communications and Networking Conference Workshops (WCNCW), pp: 69-74.

[16] TIAN Yafei, LU Songtao. \& YANG Chenyang.2013. Macro-Pico Amplitude-Space Sharing with Optimized Han-Kobayashi Codin. IEEE Transactions on Communications, 61(10): 4404-4415.

[17]GRESSET N, HALBAUERH. \& KOPPENBORG J, et al. 2012.Interference-Avoidance Techniques: Improving Ubiquitous User Experienc. IEEE Vehicular Technology Magazine,7(4): 37-45.

[18]P. A. Morreale \& J. M. Anderson. 2014. Software Defined Networking: Design and Deployment. Boca Raton, FL, USA: CRC Press.

[19]S. Sezer et al. 2013. Are we ready for SDN? Implementation challenges for software-defined networks, IEEE Commun. Mag., 51(7): 36-43.

[20] Slavica Tomovic, Milica Pejanovic-Djurisic. \& Igor Radusinovic. 2014. SDN Based Mobile Networks: Concepts and Benefits. Wireless Personal Communications, 78 (3): 1629-1644.

[21] Gao X Q, You X H. \& Jiang B, et al. 2004 MIMO-GMC wireless transmission technology for beyond $3 \mathrm{G}$ mobile communications. Acta Electron Sin, 12A: $105-108$

[22]B. Farhang-Boroujeny. 2011. OFDM Versus Filter Bank Multicarrier, IEEE Signal Processing Magazine, 28(3) 92-112.

[23] Kishiyama Y. Future radio access for 5G. NTT DOCOMO, Inc

[24] Estella I, Pascual-Iserte A. \& Payar M. 2010. OFDM and FBMC performance comparison for multistream MIMO systems. In: Proceedings of Future Network and Mobile Summit, pp: 1-8.

[25] Wunder G, Kasparick M. \& ten Brink S. 2013. 5G NOW: Challenging the LTE design paradigms of orthogonality and synchronicity. In: Proceedings of IEEE Vehicular Technology Conference (VTC Spring), pp: $1-5$.

[26]Pinchon D. \& Siohan P. 2013. Derivation of analytical expressions for flexible PR low complexity FBMC Systems. In: Proceedings of European Signal Processing Conference, pp: 1-5.

[27] Sahin A, Guvenc I, Arslan H. A survey on multicarrier communications: prototype filters, lattice structures, and implementation Http://arxiv.org/abs/1212.3374v2 